\title{
The procedure for intraoperative graft verification in coronary surgery: Is high resolution epicardial imaging useful in addition to Transit-Time flow measurement to reduce postoperative failures?
}

\author{
Gabriele Di Giammarco*, Daniele Marinelli, Massimiliano Foschi, Tariq Mobariki, Donato Micucci, \\ Francesca Faragalli, Michele Di Mauro
}

From World Society of Cardiothoracic Surgeons 25th Anniversary Congress, Edinburgh Edinburgh, UK. 19-22 September 2015

\section{Background/Introduction}

Transit-Time Flow Measurement (TTFM) is a valid method of intraoperative graft verification. TTFM shows a low diagnostic accuracy due to high number of false positive.

High-Resolution Epicardial Ultrasonography (HRECUS) is a well-recognized procedure for coronary anastomosis and stenosis evaluation with high sensitivity and specificity.

\section{Aims/Objectives}

The aim of the study is to verify if the combined use of TTFM and HR-ECUS in intraoperative graft verification process can increase the diagnostic accuracy of procedure itself.

\section{Method}

From November 2009 to December 2014, 741 patients underwent isolated CABG. A total number of 1733 grafts were performed; all the grafts were intraoperatively verified by means of both TTFM and HR-ECUS.

\section{Results}

Among all grafts considered functioning at TTFM, $7(0.4 \%)$ were failing at HR-ECUS and promptly redone. These were confirmed as true positive at graft revision due to technical error. HR-ECUS confirmed the good functioning of the remaining grafts already demonstrated by TTFM; among them, 8 showed high troponin
I release (clinical false negative), whereas the remaining had no high TnI release (clinical true negative). In 2 of 39 grafts malfunctioning at TTFM, HR-ECUS confirmed the graft failure. Finally, in 35 cases, HR-ECUS did not confirm TTFM diagnosis demonstrating a full patency of the anastomosis; these grafts had an uneventful clinical course (true negative). The main result of this study is the increase of PPV from 10\% with TTFM to almost $100 \%$ of TTFM + HR-ECUS, avoiding many unnecessary graft revisions.

\section{Discussion/Conclusion}

In intraoperative graft verification procedure the combined use of TTFM and HR-ECUS increase diagnostic accuracy of the verification process close to $100 \%$. Both methods should be used intraoperatively in order to achieve the best outcome for the patient.

Published: 16 December 2015

doi:10.1186/1749-8090-10-S1-A279

Cite this article as: Di Giammarco et al:: The procedure for

intraoperative graft verification in coronary surgery: Is high resolution epicardial imaging useful in addition to Transit-Time flow measurement to reduce postoperative failures? Journal of Cardiothoracic Surgery 2015 10(Suppl 1):A279. 